\section{Consumo de álcool entre estudantes universitários}

\author{
Alcohol consumption by university students
}

\section{Abstract}

Consumption of alcoholic beverages is widely encouraged by the mass media, despite the related health risks. Today's students in the health fields are the professionals of tomorrow who will be providing advice and serving as role models for patients. The aim of this study was to analyze alcohol consumption and related factors among these students. A total of 608 male and female university students from Maceió, the capital of Alagoas State, Brazil, completed a self-administered questionnaire. Data analysis included Poisson regression and multinomial logistic models. Prevalence of lifetime use of alcohol was 90.4\%. Prevalence of alcohol abuse was $18.3 \%$ in men and $6.1 \%$ in women. Heavier alcohol consumption and alcohol abuse were observed in males, older students, non-natives of Maceió, smokers, and those exposed to alcohol advertising. The results emphasized the vulnerability of these young people to risky health behaviors. Their future social role highlights distinct needs in their university education to enable them to act professionally in this area.

Alcohol Drinking; Students; Behavior
Adriano Antonio da Silva Pedrosa 1

Luiz Antonio Bastos Camacho 2

Sônia Regina Lambert Passos 3

Raquel de Vasconcellos Carvalhaes de Oliveira ${ }^{3}$

\section{Introdução}

O uso de substâncias psicoativas, sobretudo de álcool, encontra-se presente em anúncios comerciais, filmes, letras de música e outros meios de comunicação de massa. A apresentação dessas substâncias associadas a fatores desejáveis como prazer, beleza, sucesso financeiro e sexual, poder e outros, de forma explícita ou implícita, configura-se um importante fator de risco para o seu consumo abusivo ${ }^{1}$. Além disso, o consumo de bebidas alcoólicas pode estar associado ao consumo de tabaco e ao comportamento sexual de risco, os quais são alguns dos principais fatores relacionados com o estado de saúde dos indivíduos e das populações.

O consumo de bebidas alcoólicas como acompanhamento da alimentação e de celebrações tem sido observado em diferentes civilizações e culturas na história da Humanidade. O grande papel que a produção e o consumo de bebidas alcoólicas desempenham na vida social e econômica das sociedades ocidentais não deve permitir que se menospreze o fato de que o problema do alcoolismo é muito maior do que todas as outras formas combinadas do abuso de substâncias 2 .

A experiência universitária é única, pois dá aos estudantes a primeira oportunidade de ser parte de um grande grupo de pares sem supervisão familiar. Isto os torna mais vulneráveis a 
tentar romances, experiências previamente proibidas e algumas vezes ilícitas ${ }^{3}$.

Alguns estudos sobre o diagnóstico de abuso ou dependência de álcool em estudantes têm sido realizados no Brasil, particularmente nas regiões Sudeste e Sul 2,4,5,6,7,8,9,10,11,12. No que concerne especificamente ao uso de substâncias no Brasil, pesquisas revelam que o álcool é a substância mais consumida pelos jovens, seguida pelo tabaco, maconha e estimulantes 13; o que pode representar um fator de risco para a adoção de outros comportamentos de risco à saúde, tais como beber e dirigir, atividade sexual desprotegida, violência e suicídio 14,15,16.

Estudos sobre o uso de substâncias psicoativas têm mostrado um panorama ascendente para o consumo de álcool em adolescentes e jovens 17. Conforme relatam Andrade et al. $14 \mathrm{e}$ Kerr-Corrêa et al. ${ }^{10}$, o álcool foi apontado como a substância mais utilizada por estudantes da Universidade de São Paulo (USP) e da Universidade Estadual Paulista (UNESP), na cidade e no estado de São Paulo, respectivamente. No Chile, o alcoolismo também constitui problema relevante e afeta especialmente a população de jovens 18 . O consumo de álcool no Reino Unido aumentou entre as idades de 18 e 20 anos e há evidências de que muitos estão bebendo de maneira potencialmente prejudicial para a saúde 19 .

Levando em consideração todos os aspectos comentados, evidencia-se a importância de ampliar os estudos sobre este tema em estudantes de regiões brasileiras fora do eixo Sudeste e Sul. Com o presente estudo, se pretende determinar o perfil epidemiológico do consumo de álcool e fatores relacionados entre os estudantes de ensino superior da área de saúde em uma cidade da Região Nordeste. Espera-se que os resultados deste estudo contribuam para dimensionar o problema e que forneçam elementos para propor ações de controle.

\section{Métodos}

Foram incluídos neste estudo os estudantes matriculados nas Faculdades das Ciências da Saúde (Medicina, Enfermagem, Odontologia, Nutrição, Farmácia e Fisioterapia) de duas universidades públicas alagoanas, no ano de 2002, localizadas em Maceió, capital do estado de Alagoas. Maceió está localizada no nordeste do país e tem uma população de 936.314 habitantes. As duas universidades que sediaram a pesquisa, uma federal e a outra estadual, eram as únicas na área da saúde, na época.

É um estudo seccional, de caráter descritivo e analítico, sobre a quantidade e frequência de consumo de álcool e tabaco e o grau de informação sobre questões de saúde relacionadas ao uso de álcool, tabaco e comportamento sexual. O tamanho amostral necessário foi calculado para um nível de $5 \%$ de significância, erro amostral de $5 \%$, prevalência de $50 \%$, correção para população finita e efeito de desenho de 1,2 ( $\mathrm{n}=383)$. Ao tamanho amostral calculado foram acrescidos $10 \%$ por possíveis perdas, o que resultou em 422 estudantes. Foi realizada uma amostragem aleatória por conglomerados em um estágio, onde o conglomerado foi a turma de alunos, previamente estratificada por curso e ano acadêmico. A taxa de não participação, principalmente pela ausência no dia da aplicação do questionário, variou, em média, de 32,4\% (Farmácia) a 59,2\% (Enfermagem) e de $35,2 \%$ ( $1^{\circ}$ ano) a $70,5 \%$ (6o ano). A maior ausência dos estudantes dos últimos anos da graduação se deve à sua disponibilidade limitada, especialmente pela distribuição fragmentada em diversos setores (enfermarias, ambulatórios, hospitais etc.). Ao final, dos 1.830 estudantes universitários elegíveis, alcançamos uma amostra de 608 estudantes, representando a grande mobilização e adesão dos estudantes participantes.

Os dados foram coletados por monitores da disciplina de Saúde Coletiva da Universidade Federal de Alagoas (UFAL), capacitados e treinados para aplicação dos questionários, esclarecimentos de eventuais dúvidas dos participantes e soluções quanto a possíveis intercorrências, coordenados pelo pesquisador responsável pela pesquisa. O questionário administrado foi adaptado do que era utilizado no Departamento de Sanidade e Seguridade Social da Generalitat de Catalunya (Espanha) em estudos populacionais 20,21. É um questionário anônimo, de autopreenchimento, não validado no Brasil, que apresenta uma linguagem de nível compatível com estudantes de ensino superior, que pretende avaliar os comportamentos e conhecimentos dos estudantes universitários com respeito ao consumo de tabaco e álcool, a prática de atividades físicas e a sexualidade. Os itens mais sensíveis do instrumento levaram em conta uma enorme experiência já acumulada e disponível na literatura sobre as abordagens válidas ${ }^{21}$, de maneira que não se pretendeu inovar em perguntas sobre consumo de álcool, tabaco e comportamento sexual. Para o abuso de álcool, por exemplo, utilizamos o parâmetro de doses/semana sugerido pela Organização Mundial da Saúde (OMS) 22, e consideramos, assim como em outros itens do questionário, sua validade de conteúdo.

O consumo de álcool foi aferido através de questões sobre o consumo das seguintes bebidas alcoólicas: cerveja, vinho, vinho doce, champa- 
nhe, vermutes, brandy/conhaque, uísque, aguardentes, licores, combinados e outras.

Para as bebidas alcoólicas, os estudantes informaram a quantidade e a frequência de consumo no último ano, tipificada em seis categorias: diariamente, semanalmente, mensalmente, anualmente, bebeu alguma vez (há mais de 12 meses) e nunca bebeu (abstêmio). Definiu-se o "uso na vida" de consumo de álcool como o consumo de "bebida alcoólica pelo menos uma vez em sua vida”. A partir das respostas do questionário se calculou o consumo total diário e semanal em equivalentes de álcool puro (etanol) em gramas e em unidades-padrão ( $10 \mathrm{~g}$ de álcool = 1 unidade-padrão), diferenciando o consumo durante a semana (de segunda a quinta-feira) e durante o fim de semana (de sexta-feira a domingo). $\mathrm{O}$ consumo total de álcool foi categorizado posteriormente em quatro faixas: nenhum consumo; até 14 unidades/semana; entre 15 e 21 unidades/ semana; e mais de 21 unidades/semana. Ditos limites se fixaram atendendo aos níveis de risco descritos na literatura para a população igual ou maior de 18 anos: consumo de mais de 21 unidades/semana para os homens e mais de 14 unidades/semana para as mulheres 22 , os quais caracterizam o abuso de álcool.

As variáveis quantitativas contínuas foram descritas através da média (e desvio-padrão), ou mediana (e valores mínimo e máximo) se apresentassem distribuição assimétrica. Para as variáveis qualitativas nominais e ordinais usamos a descrição dos percentuais e valores absolutos. As análises bivariadas entre as variáveis qualitativas (sexo, idade, curso, ano acadêmico, lugar de nascimento, tabagismo, comportamento sexual de risco para gravidez não planejada e doenças sexualmente transmissíveis) e o abuso de álcool (sim/não) foram investigadas pelo teste quiquadrado de Pearson, ao nível de significância de 5\%. Razões de prevalência não ajustadas (RP) foram calculadas para avaliar a magnitude das associações.

Foram realizadas regressões de Poisson e logística multinomial, com os seguintes desfechos, respectivamente: prevalência de uso na vida e de uso abusivo de álcool e o consumo do álcool classificado em três categorias (abstêmio, uso e abuso). As covariáveis utilizadas nos dois modelos de regressão foram aquelas avaliadas na análise bivariada, que apresentaram valor de $\mathrm{p} \leq 0,20$. As variáveis que ficaram no modelo final foram aquelas que apresentaram valor de $\mathrm{p}<0,05$. Algumas variáveis foram construídas a partir de um conjunto de itens do questionário, dicotomizando-as para inclusão nos modelos de regressão. Os dados foram registrados e analisados nos programas estatísticos SPSS versão 16.0 (SPSS Inc.,
Chicago, Estados Unidos), R versão 2.9.1 (The R Foundation for Statistical Computing, Viena, Áustria; http://www.r-project.org) e WinPepi versão 7.6 (http://www.brixtonhealth.com/pe pi4windows.html). Todas as análises estatísticas consideraram o peso e o plano amostral.

Esta pesquisa foi submetida e aprovada pelos Comitês de Ética em Pesquisa da UFAL (parecer 006822/2002-19), da Universidade Estadual de Ciências da Saúde de Alagoas (UNCISAL; parecer 085/2002) e da Escola Nacional de Saúde Pública Sergio Arouca, Fundação Oswaldo Cruz (ENSP/ Fiocruz; parecer 88/09). A Fundação de Amparo à Pesquisa do Estado do Rio de Janeiro (FAPERJ) forneceu suporte financeiro a este estudo.

\section{Resultados}

A maioria dos 608 estudantes $(78,3 \%)$ era do sexo feminino, com média de 21,3 anos de idade $(\mathrm{DP}=2,5)$ e natural de Maceió $(51 \%)$. Pouco mais da metade cursava Medicina ou Enfermagem $(55,6 \%)$ e cerca de dois terços $(66,7 \%)$ eram dos três primeiros anos de cada curso, à exceção de Medicina que apresentava mais alunos distribuídos ao longo de mais anos acadêmicos (Tabela 1). A taxa de resposta variou de $40,8 \%$ (Enfermagem) a $67,6 \%$ (Farmácia) e de $29,5 \%$ (6º ano) a $64,8 \%$ (1o ano).

Quase todos os estudantes mostraram ter conhecimentos sobre a transmissão sexual de algumas doenças, entretanto, um percentual razoável não conhecia as doenças sexualmente transmissíveis perguntadas $(79,9 \%)$ nem os métodos anticoncepcionais $(64,1 \%)$.

O uso de tabaco em alguma fase da vida foi referido por 169 (27,8\%) dos participantes. A média de idade de experimentação foi 15,1 anos $(\mathrm{DP}=2,8)$. Entre os fumantes, a média de cigarros consumidos foi de 15,7 durante a semana e 12,2 no fim de semana, o que representa 3,9 (DP = 4,9) e 4,1 ( DP = 3,6) cigarros/dia, respectivamente (dados não mostrados).

A prevalência de uso de álcool na vida foi de $90,4 \%$. A grande maioria $(94,9 \%)$ dos estudantes relatou ter visto publicidade sobre bebidas alcoólicas e $27,2 \%$ deles relataram ter consumido alguma bebida alcoólica por ter visto anunciada em alguma propaganda. Estes anúncios publicitários de bebidas alcoólicas foram vistos principalmente pelos estudantes universitários na televisão (94,6\%) e jornais e/ou revistas (82,7\%). As bebidas mais consumidas devido à publicidade foram cerveja $(57,6 \%)$, combinados $(40 \%)$ e champanhe (30,3\%) (Tabela 2).

Mais de dois terços dos estudantes $(69,2 \%)$ viram campanhas ou outras atividades contra 
Dados sociodemográficos, consumo de álcool e tabaco e fatores associados dos 608 estudantes das Ciências da Saúde de universidades públicas de Maceió, Alagoas, Brasil, 2002.

\begin{tabular}{|c|c|c|}
\hline Variável & $\mathrm{n}$ & $\%$ \\
\hline \multicolumn{3}{|l|}{ Sexo } \\
\hline Feminino & 476 & 78,3 \\
\hline Masculino & 132 & 21,7 \\
\hline \multicolumn{3}{|l|}{ Idade (anos) } \\
\hline $17-20$ & 224 & 37,0 \\
\hline $21-24$ & 336 & 55,4 \\
\hline $25 e+$ & 46 & 7,6 \\
\hline \multicolumn{3}{|l|}{ Naturalidade } \\
\hline Maceió & 310 & 51,2 \\
\hline Interior de Alagoas & 138 & 22,8 \\
\hline Outras cidades & 157 & 26,0 \\
\hline \multicolumn{3}{|l|}{ Curso } \\
\hline Medicina & 253 & 41,6 \\
\hline Enfermagem & 85 & 14,0 \\
\hline Odontologia & 87 & 14,3 \\
\hline Nutrição & 73 & 12,0 \\
\hline Farmácia & 59 & 9,7 \\
\hline Fisioterapia & 51 & 8,4 \\
\hline \multicolumn{3}{|l|}{ Ano acadêmico } \\
\hline $1 ㅇ$ & 147 & 24,2 \\
\hline 20 & 117 & 19,2 \\
\hline 3으 & 142 & 23,3 \\
\hline 4으 & 119 & 19,6 \\
\hline 5은 & 60 & 9,9 \\
\hline 60 & 23 & 3,8 \\
\hline \multicolumn{3}{|l|}{ Consumo de álcool } \\
\hline Abstêmio (Sim) & 59 & 9,6 \\
\hline Uso na vida (Sim) & 549 & 90,4 \\
\hline Uso atual (Sim) & 496 & 81,6 \\
\hline Abuso (Sim) & 53 & 8,7 \\
\hline Tabagismo (Sim) & 169 & 27,8 \\
\hline Conhece os efeitos do tabaco (Não) & 489 & 81,0 \\
\hline Recebeu conselho médico * (Sim) & 16 & 3,3 \\
\hline Adequação da abordagem do álcool (Não) & 393 & 65,6 \\
\hline Viu campanhas contra o álcool (Não) & 184 & 30,5 \\
\hline Viu publicidade do álcool (Sim) & 575 & 94,6 \\
\hline Etilismo familiar (Sim) & 308 & 51,9 \\
\hline Conhece os efeitos do álcool (Não) & 367 & 60,7 \\
\hline Conhece as DSTs (Não) & 482 & 79,9 \\
\hline Conhece a AIDS (Não) & 337 & 55,4 \\
\hline Conhece os métodos anticoncepcionais (Não) & 388 & 64,1 \\
\hline Usa método contraceptivo confiável (Não) & 34 & 5,6 \\
\hline
\end{tabular}

*Apenas os que bebiam pelo menos algumas vezes ao ano $(N=489)$. 
Bebidas alcoólicas consumidas, exposição à publicidade e campanhas de álcool em estudantes das Ciências da Saúde de universidades públicas de Maceió, Alagoas, Brasil, 2002.

\begin{tabular}{|c|c|c|}
\hline & $\mathrm{n}$ & $\%$ \\
\hline \multicolumn{3}{|l|}{ Bebidas alcoólicas ( $\mathrm{N}=608$ ) } \\
\hline Cerveja & 215 & 35,4 \\
\hline Vinho & 165 & 27,1 \\
\hline Champanhe & 54 & 8,9 \\
\hline Vermutes & 53 & 8,7 \\
\hline Brandy/Conhaque & 6 & 1,0 \\
\hline Uísque & 44 & 7,2 \\
\hline Aguardentes & 38 & 6,3 \\
\hline Licores & 41 & 6,7 \\
\hline Combinados & 285 & 46,9 \\
\hline \multicolumn{3}{|c|}{ Publicidade do álcool ( $N=608)$} \\
\hline Televisão & 573 & 94,6 \\
\hline Rádio & 374 & 61,7 \\
\hline Jornais/Revistas & 501 & 82,7 \\
\hline Fôlderes/Folhetos & 206 & 34,0 \\
\hline Publicidade da rua & 447 & 73,8 \\
\hline \multicolumn{3}{|c|}{ Campanhas contra o álcool ( $N=608)$} \\
\hline Televisão & 606 & 99,8 \\
\hline Rádio & 183 & 30,1 \\
\hline Jornais/Revistas & 286 & 47,1 \\
\hline Prospectos/Folhetos & 169 & 27,8 \\
\hline Propagandas na rua & 169 & 27,8 \\
\hline Professores da faculdade & 64 & 10,5 \\
\hline Profissionais sanitários & 52 & 8,6 \\
\hline \multicolumn{3}{|c|}{ Bebidas consumidas por terem sido anunciadas $(N=165)$} \\
\hline Cerveja & 95 & 57,6 \\
\hline Vinho & 23 & 13,9 \\
\hline Champanhe & 50 & 30,3 \\
\hline Vermutes & 47 & 28,5 \\
\hline Brandy/Conhaque & 21 & 12,7 \\
\hline Uísque & 35 & 21,2 \\
\hline Aguardentes & 46 & 27,9 \\
\hline Licores & 32 & 19,4 \\
\hline Combinados & 66 & 40,0 \\
\hline
\end{tabular}

o consumo de bebidas alcoólicas. Os principais meios de comunicação relatados por estes foram: a televisão $(99,8 \%)$, os jornais e revistas $(68,1 \%)$ e o radio $(43,6 \%)$. Entre os que viram as campanhas, $22,1 \%$ apresentavam um consumo diário ou semanal de álcool, embora a frequência de consumo não tenha apresentado diferenças estatisticamente significativas.

Das 17 variáveis selecionadas na análise bivariada, apenas oito apresentaram significância estatística no modelo de Poisson para explicação do uso abusivo de álcool (sim/não), ao nível de
5\% de significância: sexo, receber conselho médico, ver publicidade do álcool, etilismo familiar, conhecimento dos efeitos do álcool, conhecer DSTs, conhecer AIDS e conhecer os métodos anticoncepcionais. A prevalência de abuso de álcool nos participantes da pesquisa foi de $8,7 \%$. Os homens apresentaram uma prevalência de uso abusivo de bebidas alcoólicas quase três vezes maior que as mulheres $\left(\mathrm{RP}_{\text {ajustada }}=2,90\right.$; $\mathrm{p}<$ 0,001) (Tabela 3). Os estudantes que receberam conselho médico para reduzir o seu consumo de bebidas alcoólicas apresentaram uma prevalên- 
Razão de prevalência (RP) bruta e ajustada do abuso de álcool (sim/não) e fatores associados em estudantes das Ciências da Saúde de universidades públicas de Maceió, Alagoas, Brasil, 2002 ( $N=608$ ).

\begin{tabular}{|c|c|c|c|c|c|}
\hline & $\mathrm{n}$ & $\%$ & $\mathrm{RP}$ & $\mathrm{RP}_{\text {ajustada }}$ * & IC95\% \\
\hline \multicolumn{6}{|l|}{ Sexo } \\
\hline Feminino & 29 & 6,1 & 1,00 & 1,00 & \\
\hline Masculino & 24 & 18,3 & 3,01 & 2,90 & $1,73-4,87$ \\
\hline \multicolumn{6}{|l|}{ Idade (anos) } \\
\hline $17-20$ & 14 & 6,3 & 1,00 & & \\
\hline $21-24$ & 31 & 9,2 & 1,52 ** & & \\
\hline $25 e+$ & 8 & 17,8 & 3,24 ** & & \\
\hline \multicolumn{6}{|l|}{ Naturalidade } \\
\hline Maceió & 25 & 8,1 & 1,00 & & \\
\hline Interior de Alagoas & 7 & 5,1 & 0,61 & & \\
\hline Outras cidades & 21 & 13,5 & 1,77 & & \\
\hline \multicolumn{6}{|l|}{ Curso } \\
\hline Medicina, Enfermagem e Odontologia & 40 & 9,4 & 1,00 & & \\
\hline Nutrição, Farmácia e Fisioterapia & 13 & 7,1 & 0,73 & & \\
\hline \multicolumn{6}{|l|}{ Ano acadêmico } \\
\hline 1으 e 2으 & 21 & 8,0 & 1,00 & & \\
\hline 3 으 e 4 으 & 24 & 9,2 & $1,18 * \star \star$ & & \\
\hline 50 e 60 & 8 & 9,6 & $1,23 * \star \star$ & & \\
\hline \multicolumn{6}{|l|}{ Tabagismo } \\
\hline Não & 45 & 8,2 & 1,00 & & \\
\hline $\operatorname{Sim}$ & 8 & 14,3 & 1,87 & & \\
\hline \multicolumn{6}{|l|}{ Conhece os efeitos do tabaco } \\
\hline $\operatorname{Sim}$ & 9 & 7,8 & 1,00 & & \\
\hline Não & 43 & 8,8 & 1,13 & & \\
\hline \multicolumn{6}{|l|}{ Recebeu conselho médico \# } \\
\hline Não & 46 & 9,8 & 1,00 & 1,00 & \\
\hline $\operatorname{Sim}$ & 7 & 43,8 & 4,48 & 5,62 & $1,94-16,29$ \\
\hline \multicolumn{6}{|l|}{ Adequação da abordagem do álcool } \\
\hline $\operatorname{Sim}$ & 15 & 7,3 & 1,00 & & \\
\hline Não & 37 & 9,4 & 1,29 & & \\
\hline \multicolumn{6}{|l|}{ Viu campanhas contra o álcool } \\
\hline Não & 13 & 7,1 & 1,00 & & \\
\hline Sim & 38 & 9,0 & 1,28 & & \\
\hline \multicolumn{6}{|l|}{ Viu publicidade do álcool } \\
\hline Não & 49 & 8,5 & 1,00 & 1,00 & \\
\hline Sim & 3 & 10,3 & 1,21 & 3,94 & $1,90-8,19$ \\
\hline \multicolumn{6}{|l|}{ Etilismo familiar } \\
\hline Sim & 22 & 7,1 & 1,00 & 1,00 & \\
\hline Não & 29 & 10,1 & 1,42 & 2,08 & $1,05-4,13$ \\
\hline \multicolumn{6}{|l|}{ Conhece os efeitos do álcool } \\
\hline Não & 24 & 6,5 & 1,00 & 1,00 & \\
\hline $\operatorname{Sim}$ & 28 & 11,8 & 1,80 & 2,01 & $1,02-3,96$ \\
\hline \multicolumn{6}{|l|}{ Conhece as DSTs } \\
\hline Não & 33 & 6,8 & 1,00 & 1,00 & \\
\hline Sim & 20 & 16,5 & 2,41 & 3,45 & $1,68-7,10$ \\
\hline
\end{tabular}




\begin{tabular}{|c|c|c|c|c|c|}
\hline & $\mathrm{n}$ & $\%$ & RP & $\mathrm{RP}_{\text {ajustada }}$ * & IC95\% \\
\hline \multicolumn{6}{|c|}{ Conhece a AIDS } \\
\hline $\operatorname{Sim}$ & 20 & 7,4 & 1,00 & 1,00 & \\
\hline Não & 33 & 9,8 & 1,33 & 2,10 & $1,20-3,68$ \\
\hline \multicolumn{6}{|c|}{ Conhece os métodos anticoncepcionais } \\
\hline Não & 32 & 8,2 & 1,00 & 1,00 & \\
\hline Sim & 21 & 9,7 & 1,17 & 2,00 & $1,08-3,69$ \\
\hline \multicolumn{6}{|c|}{ Usa método contraceptivo confiável } \\
\hline Não & 2 & 5,9 & 1,00 & & \\
\hline Sim & 51 & 8,9 & 1,52 & & \\
\hline
\end{tabular}

IC95\%: intervalo de 95\% de confiança.

* Razão de prevalência ajustada por regressão loglinear de Poisson com estimador robusto na matriz de covariâncias.

Estimativas ajustadas estão apresentadas no modelo final para as variáveis que foram estatisticamente significativas $(p<0,05)$;

** Likelihood ratio tests $\left(\chi^{2}=1922,97 ; p<0,001\right)$;

$\star \star \star$ Likelihood ratio tests $\left(\chi^{2}=210,58 ; p<0,001\right)$;

\# Apenas os que bebiam pelo menos algumas vezes ao ano $(N=489)$.

cia 4,6 vezes maior (2,0 vezes para os homens e 5,2 para as mulheres) de abusar do álcool que os que não receberam este conselho. Os homens que viram anúncios publicitários de bebidas alcoólicas apresentaram uma prevalência de abuso de álcool 2,9 vezes maior do que aqueles que não viram estes anúncios ( $\mathrm{p}<0,001)$. Os que não tiveram algum parente que padeceu de problemas derivados do álcool apresentaram chance de abuso de álcool 1,1 vez maior que os que tiveram, especialmente entre os homens $(21,8 \%$ contra $14,3 \%)$. Com relação ao comportamento sexual observou-se que aqueles que não conheciam a probabilidade de transmissão da AIDS, mas conheciam as DSTs e os métodos anticoncepcionais perguntados, apresentaram prevalências superiores de abuso de álcool (Tabela 3).

Das 17 variáveis selecionadas na análise bivariada, quatro apresentaram significância estatística $(\mathrm{p}<0,05)$ no modelo final da regressão logística multinomial (Tabela 4), que considera o uso de álcool em três categorias (abstêmios, uso e abuso). Aqueles que eram do sexo masculino, os fumantes, os que não conheciam todos os efeitos do tabaco, os que viram as campanhas contra o álcool e aqueles que não tiveram parentes com problemas derivados do álcool apresentaram maiores chances de consumir álcool (uso ou abuso).

\section{Discussão}

A prevalência de consumo na vida de álcool foi bastante alta e semelhante à encontrada em outros estudos 5,13,14,16,17,22,23, destacando a tendên- cia ascendente para o consumo em adolescentes e jovens. Em 2005, o uso na vida de álcool nas 107 maiores cidades do país foi de $74,6 \%$, porcentagem maior que em 2001 com 68,7\% 16. Nesta amostra de universitários, a periodicidade também foi alta, especialmente entre os homens, entre os quais $42 \%$ relataram beber pelo menos uma vez por semana. As bebidas alcoólicas mais consumidas também seguiram o padrão encontrado em outros estudos, onde os homens preferem a cerveja e as mulheres os combinados e o vinho 11,12 . Intuitivamente, o clima, o preço e fatores culturais poderiam explicar as preferências pelo tipo de bebida.

O abuso de álcool neste estudo $(8,7 \%)$ foi bem menor que o encontrado em estudantes universitários britânicos 23 (25\% a 42\%), semelhante ao de estudantes de medicina de universidades americanas públicas e privadas 24 (6\% a $12 \%)$ e maior que em estudantes de medicina de Creta, Grécia 25 (3,6\% a 5,2\%). No entanto o abuso de álcool diferiu segundo o sexo atingindo 18,3\% em homens, resultado semelhante ao descrito aplicando-se o instrumento CAGE a estudantes de medicina brasileiros, em $20055^{5}$. Medidas preventivas devem considerar que este hábito é atribuído parcialmente ao estresse relacionado a novas responsabilidades específicas das atribuições destas profissões de saúde e como estes estudos citados foram realizados em universitários de cursos das Ciências da Saúde, com metodologia semelhante à realizada em nosso estudo, isto nos leva a acreditar que as diferenças observadas se devem a diferenças socioculturais.

A maioria dos estudos realizados no final da década de 90 e início dos anos 2000 4,13,15,25 mos- 
Odds ratios (OR) ajustados do consumo de álcool *,** (abstêmio, uso e abuso) e fatores associados em estudantes das Ciências da Saúde de universidades públicas de Maceió, Alagoas, Brasil, em 2002 (N = 608).

\begin{tabular}{|c|c|c|c|c|}
\hline & \multicolumn{2}{|c|}{ Uso } & \multicolumn{2}{|c|}{ Abuso } \\
\hline & $\mathrm{OR} *, \star \star$ & IC95\% & $\mathrm{OR} *, * \star$ & IC95\% \\
\hline \multicolumn{5}{|l|}{ Sexo } \\
\hline Feminino & 1,00 & & 1,00 & \\
\hline Masculino & 1,40 & $0,83-2,36$ & 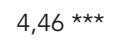 & $2,13-9,33$ \\
\hline \multicolumn{5}{|l|}{ Idade (anos) } \\
\hline $17-20$ & 1,00 & & & \\
\hline $21-24$ & 0,62 & $0,38-1,03$ & 0,92 & $0,38-2,22$ \\
\hline $25 e+$ & $0,36 * \star \star$ & $0,16-0,84$ & 1,02 & $0,29-3,66$ \\
\hline \multicolumn{5}{|c|}{ Ano acadêmico } \\
\hline 1 으 e 2 으 & 1,00 & & 1,00 & \\
\hline 3으 e 4으 & $1,81 * \star \star$ & $1,11-2,95$ & 1,42 & $0,62-3,24$ \\
\hline $5 \circ$ e 60 & $3,03 * \star *$ & $1,43-6,42$ & 1,48 & $0,45-4,94$ \\
\hline \multicolumn{5}{|l|}{ Tabagismo } \\
\hline Sim & $3,78^{* * *}$ & $1,43-9,99$ & $4,61 * \star \star$ & $1,34-15,80$ \\
\hline \multicolumn{5}{|c|}{ Conhece os efeitos do tabaco } \\
\hline Não & $2,20 * * \star$ & $1,36-3,57$ & $2,90 * * *$ & $1,20-7,03$ \\
\hline \multicolumn{5}{|c|}{ Adequação da abordagem do álcool } \\
\hline Não & $0,58 * \star \star$ & $0,37-0,90$ & 0,79 & $0,37-1,69$ \\
\hline \multicolumn{5}{|c|}{ Viu campanhas contra o álcool } \\
\hline Não & $0,63 * \star \star$ & $0,41-0,94$ & 0,60 & $0,28-1,28$ \\
\hline \multicolumn{5}{|c|}{ Etilismo familiar } \\
\hline Sim & $0,66 * * *$ & $0,45-0,97$ & 0,55 & $0,28-1,08$ \\
\hline \multicolumn{5}{|c|}{ Conhece as DSTs } \\
\hline Não & 1,07 & $0,63-1,82$ & $0,34 * \star \star \star$ & $0,15-0,76$ \\
\hline
\end{tabular}

* Categoria de referência: abstêmios;

** Ajustada por regressão logística multinomial com estimador robusto na matriz de covariâncias. Estimativas ajustadas estão apresentadas no modelo final para as variáveis que foram estatisticamente significativas $(p<0,05)$;

${ }^{\star \star \star} p<0,05$.

travam prevalências de tabagismo bem maiores que a encontrada em nosso estudo (variando de $40 \%$ a $65 \%$, em estudos nacionais, e de $30 \%$ a $90 \%$, em estudos internacionais). Em 2005, o uso na vida de tabaco teve uma prevalência de $44 \%$ da população entrevistada nas 107 maiores cidades do país, ao passo que no levantamento domiciliar de 2001 foi de 41,1\% 16,26. A prevalência foi maior na Região Sul do Brasil (49,3\% em 2005 e 44,1\% em 2001, indicando pequena heterogeneidade regional. Estudo anterior em 1.054 estudantes de Medicina no Rio de Janeiro evidenciou um uso na vida de tabaco bem maior (54,3\%) 5 . A baixa prevalência de tabagismo encontrada em nosso estudo confirma a tendência decrescente vista em alguns estudos realizados em estudantes universitários 11,18, que poderia ser atribuída, em parte, ao êxito da política brasileira para combater o tabagismo. Em que pese o menor consumo, o tabagismo esteve associado aos desfechos de uso e abuso de álcool, sugerindo a pertinência de reforçar mensagens preventivas sobre o tabaco nesta população específica.

O reconhecimento da transmissão de DST foi alto e semelhante ao encontrado em outros estudos 27,28. A desinformação sobre a AIDS, mas não sobre DST e anticoncepcionais, foi mais frequente entre os que abusavam de álcool, o que potencializa riscos, uma vez que o abuso de álcool na literatura correlaciona-se com práticas sexuais não seguras 15,27 .

O abuso do álcool esteve associado à exposição à publicidade do álcool em muitos meios de comunicação, e sua influência no consumo de bebidas alcoólicas foi demonstrada também em outros estudos 26,29. Esta literatura sobre a influência da publicidade é ainda muito escassa, o que nos incita a realizar mais estudos que 
investiguem estas questões, elucidando suas consequências e indicando ações de controle. A restrição ou a abolição deste tipo de publicidade como parte da estratégia de redução do abuso do álcool, precisam ser graduais para acomodar interesses econômicos muito grandes e diversos, assim como foi, e continua sendo de outras formas, para o tabaco. Medidas legislativas recentes, tais como a Lei no.11.705 (Lei Seca) 30, têm demonstrado sua eficácia em reduzir os acidentes de trânsito associados ao uso de álcool, alterando de certa forma o comportamento da população, com ações de persuasão combinadas com medidas de coerção.

Além de representar uma etapa importante e cheia de peculiaridades, a universidade muitas vezes leva os estudantes a residirem em outras cidades, estados e até mesmo países, o que pode torná-los mais vulneráveis. Nosso estudo revelou que estudantes vindos de fora de Maceió apresentaram um maior abuso de álcool (RP não ajustada $=$ 1,77), o que também deve ser abordado preventivamente, talvez com maior suporte da universidade a este estudante ou uma maior interação entre a universidade, a família e o estudante.

Os fatores limitantes desse estudo foram a não realização de um estudo piloto e a não validação do questionário utilizado. Tratando-se de questões sensíveis de comportamento e atitudes, poderia ter havido viés de classificação com subestimação da prevalência de abuso de álcool, por exemplo. Há evidências da validade da informação sobre o consumo de substâncias psicoativas mediante coleta de dados de maneira autodeclarada 31, o que dá aos nossos dados uma maior credibilidade e coerência. O grau de adesão que nos permitiu ampliar o tamanho da amostra sugere que o conteúdo das questões não afetou substancialmente a participação. Mesmo que entre as recusas a proporção de abusadores de álcool tenha sido maior do que nos participantes, e que as estimativas obtidas tenham sido conservadoras, consideramos que as conclusões do estudo não serão alteradas. Por fim, não estavam disponíveis dados para classificação em dependentes do álcool e da nicotina.

Apesar das limitações acima, os resultados deste estudo nos deram os elementos que permitiram uma visão crítica do consumo de álcool e tabaco e dos conhecimentos sobre DST destes universitários. A maioria dos estudos sobre uso e abuso de álcool em universitários se concentram nas regiões Sul e Sudeste restringindo a generalização dos achados (validade externa) para outros universitários das distintas regiões do Brasil. Nossos resultados permitem que futuros estudos realizados em universitários da Região Nordeste possam ser comparados aos aqui encontrados.
Adultos jovens são geralmente considerados mais propensos a práticas de risco para a saúde, especialmente os problemas relacionados ao consumo, abuso e dependência de álcool 32 . Talvez mais ainda, se os efeitos nocivos não forem de curto prazo ou se os limites entre o abuso e o consumo moderado, seguro e socialmente aceito não forem bem demarcados. Em muitas sociedades modernas esta é também a fase da vida de acelerado amadurecimento pessoal, de mudanças de laços sociais e de avanços na formação profissional, e por isso, possivelmente mais suscetível, a ações de promoção de saúde. Nesse sentido, os dados deste estudo adquirem significado especial, supondo que o nível socioeconômico e o acesso à informação diferenciem os estudantes universitários da área da saúde, dos demais jovens.

Os grupos que apresentaram maior consumo de álcool, especialmente o abuso de álcool, foram os estudantes universitários do sexo masculino, os de maior idade, os fumantes e aqueles que estavam expostos à publicidade do álcool, devem ser os alvos das medidas preventivas seja com mudanças na abordagem curricular do tema em disciplinas, seminários e pesquisas além de campanhas específicas nos ambiente universitário; como também fora dele, através de campanhas publicitárias para jovens e atividades extracurriculares em instituições que prestem assistência aos dependentes alcoólicos. Devemos repensar o impacto que as mensagens publicitárias contra o consumo de álcool têm alcançado nestes estudantes, com revisão aprofundada sobre o conteúdo das mesmas, e com nível de sofisticação comparável ao das mensagens dos fabricantes.

Os resultados deste estudo indicam uma vulnerabilidade destes indivíduos para condutas com risco para a saúde. O papel social futuro destinado a estes jovens suscita necessidades distintas de formação universitária para que possam atuar profissionalmente nesta área de acordo com suas especificidades. Considerando que este grupo de universitários da área da saúde possivelmente tem maior facilidade de entendimento das implicações do uso e abuso de álcool, do tabagismo e do comportamento sexual, a abordagem destas questões suscita necessidades distintas. Nesse sentido, a universidade apresenta uma grande diversidade de oportunidades, que vão além das disciplinas e incluem outras atividades acadêmicas. No médio prazo ações eficazes de promoção da saúde sobre esta população universitária podem impactar toda a população futuramente atendida por eles em unidades de saúde naquela região geográfica. 


\section{Resumo}

O consumo de álcool é estimulado pela mídia apesar dos riscos inerentes à saúde. Estudantes da área de saúde serão os futuros profissionais a orientar a população ou servir de exemplo. O objetivo deste estudo foi analisar o consumo de álcool e fatores relacionados nestes estudantes. Participaram 608 estudantes universitários de Maceió, Alagoas, Brasil, de ambos os sexos, mediante a aplicação de um questionário autopreenchivel. Foram empregados modelos de regressão de Poisson e logística multinomial. A prevalência de uso na vida de álcool foi de 90,4\%. O abuso de álcool teve uma prevalência de 18,3\% nos homens e 6,1\% nas mulheres. Os que apresentaram maior consumo e abuso de álcool foram os do sexo masculino, de maior idade, naturais de outras cidades, fumantes e os expostos à publicidade do álcool. Os resultados deste estudo indicam uma vulnerabilidade destes jovens para condutas com risco para a saúde. Seu papel social futuro suscita necessidades distintas de formação universitária para que possam atuar profissionalmente nesta área.

Consumo de Bebidas Alcoólicas; Estudantes; Comportamento

\section{Colaboradores}

A. A. S. Pedrosa participou da aplicação dos questionários, digitação e verificação da qualidade dos dados coletados, análise dos dados, discussão, conclusão e elaboração do manuscrito. L. A. B. Camacho colaborou na análise dos dados e na elaboração do manuscrito. S. R. L. Passos contribuiu com a concepção e delineamento do estudo, análise dos dados, interpretação e discussão dos resultados, redação do manuscrito e revisão da versão final. R. V. C. Oliveira contribuiu com a análise estatística dos dados, interpretação e discussão dos resultados, redação do manuscrito e revisão da versão final.

\section{Agradecimentos}

Agradecemos aos professores e monitores da UFAL e da UNCISAL, aos estudantes que participaram deste estudo, ao professor André Reynaldo Santos Périssé e a todos os demais professores e alunos participantes da Oficina de Artigos da ENSP/Fiocruz por suas contribuições. À FAPERJ.

\section{Referências}

1. Zaslow MJ, Takanishi R. Priorities for research on adolescent development. Am Psychol 1993; 48: 185-92.

2. Paduani GF, Barbosa GA, Morais JCR, Pereira JCP, Almeida MF, Prado MM, et al. Consumo de álcool e fumo entre os estudantes da Faculdade de Medicina da Universidade Federal de Uberlândia. Rev Bras Educ Méd 2008; 32:66-75.

3. Tapert S, Aarons G, Sedlar G, Brown S. Adolescent substance use and sexual risk-taking behavior. J Adolesc Health 2001;28:181.

4. Stempliuk VA, Barroso LP, Andrade AG, Nicastri S, Malbergier A. Comparative study of drug use among undergraduate students at the University of São Paulo - São Paulo campus in 1996 and 2001. Rev Bras Psiquiatr 2005; 27:185-93.

5. Lambert-Passos SR, Brasil PEAA, Santos MAB, Aquino MTC. Prevalence of psychoactive drug use among medical students in Rio de Janeiro. Soc Psychiatry Psychiatr Epidemiol 2006; 41:989-96.
6. Borini P. Influência do curso médico sobre os estudantes de medicina quanto ao uso de bebida alcoólica e sobre as atitudes, conceitos e concepções etiológicas relativos ao uso abusivo de álcool. J Bras Psiquiatr 1996; 45:703-8.

7. Borini P, Oliveira CM, Martins MG, Guimarães RC. Padrão de uso de bebidas alcoólicas de estudantes de medicina (Marília, São Paulo) - parte 1. J Bras Psiquiatr 1994; 43:93-103.

8. Borini P, Oliveira CM, Martins MG, Guimarães RC. Conceitos, concepções etiológicas e atitudes de estudantes de medicina sobre o uso e abuso de álcool: correlações com os padrões de uso - parte 2. J Bras Psiquiatr 1994; 43:123-31.

9. Mesquita AM, Laranjeira R, Dunn J. Psychoactive drug use by medical students: a review of the national and international literature. São Paulo Med J 1997; 115:1356-65. 
10. Kerr-Corrêa F, Andrade AG, Bassit AZ, Boccuto NMVF. Uso de álcool e drogas por estudantes de medicina da UNESP. Rev Bras Psiquiatr 1999; 21:95-100

11. Mardegan PS, Souza RS, Buaiz V, Siqueira MM. Uso de substâncias psicoativas entre estudantes de enfermagem. J Bras Psiquiatr 2007; 56:260-6.

12. Wagner GA, Stempliuk VA, Zilberman ML, Barroso LP, Andrade AG. Alcohol and drug use among university students: gender differences. Rev Bras Psiquiatr 2007; 29:123-9.

13. Chiapetti N, Serbena CA. Uso de álcool, tabaco e drogas por estudantes da área da saúde de uma universidade de Curitiba. Psicol Reflex Crít 2007; 20:303-13.

14. Andrade AG, Bassit AZ, Kerr-Corrêa F, Tonhon AA, Boscovitz EP, Cabral M, et al. Fatores de risco associados ao uso de álcool e drogas na vida, entre estudantes de medicina do Estado de São Paulo. Rev ABP-APAL 1997; 19:117-26.

15. Franca C, Colares V. Comparative study of health behavior among college students at the start and end of their courses. Rev Saúde Pública 2008; 42:1-7.

16. Galduróz JCF, Noto AR, Nappo SA, Carlini EA. Uso de drogas psicotrópicas no Brasil: pesquisa domiciliar envolvendo as 107 maiores cidades do país -2001. Rev Latinoam Enferm 2005; 13 Esp:888-95.

17. Gigliotti A, Bessa MA. Síndrome de dependência do álcool: critérios diagnósticos. Rev Bras Psiquiatr 2004; 26 Suppl I:S11-3.

18. Baskin-Sommers A, Sommers I. The co-occurrence of substance use and high-risk behaviors. J Adolesc Health 2008; 38:609-11.

19. Sly D, Quadagno D, Harrison D, Eberstein I, Riehman $\mathrm{K}$. The association between substance use, condom use and sexual risk among low-income women. Fam Plann Perspect 1997; 29:132-6.

20. Departament de Sanitat i Seguretat Social. Enquesta de tabaquisme en els col. lectius exemplars de l'àmbit sanitari (metges, farmacéutics i infermeres). Barcelona: Generalitat de Catalunya; 1990.

21. Prat-Marin A, Fuentes-Almendras MM, SanzGallen P, Canela-Argues R, Canela-Soler J, PardellAlenta $\mathrm{H}$, et al. Epidemiología del tabaquismo en los estudiantes de ciencias de la salud. Rev Saúde Pública 1994; 28:100-6.

22. Royal College of Psychiatrists. Alcohol: our favourite drug. London: Tavistock; 1986.

23. Newbury-Birch D, White M, Kamali J. Factors influencing alcohol and illicit drug use amongst medical students. Drug Alcohol Depend 2000; 59:125-30.
24. Webb E, Ashton CH, Kelly P, Kamali F. An update on British medical students lifestyles. Med Educ 1998; 32:325-31.

25. Mammas IN, Bertsias GK, Linardakis M, Tzanakis NE, Labadarios DN, Kafatos AG. Cigarette smoking, alcohol consumption, and serum lipid profile among medical students in Greece. Eur J Public Health 2003; 13:278-82.

26. Centro Brasileiro de Informações Sobre Drogas Psicotrópicas. I levantamento domiciliar nacional sobre o uso de drogas psicotrópicas - parte A: estudo envolvendo as 24 maiores cidades do Estado de São Paulo - 1999. http://200.144.91.102/ cebridweb/conteudo.aspx?cd=560 (acessado em Nov/2008).

27. American College Health Association. American College Health Association National College Health Assessment (ACHA-NCHA) Spring 2005 Reference Group Data Report (Abridged). J Am Coll Health 2006; 55:5-16.

28. Ma Q, Ono-Kihara M, Cong L, Xu G, Zamani S, Ravari SM, et al. Sexual behavior and awareness of Chinese university students in transition with implied risk of sexually transmitted diseases and HIV infection: a cross-sectional study. BMC Public Health 2006; 6:232.

29. Noto AR, Baptista MC, Faria ST, Nappo SA, Galduróz JCF, Carlini EA. Drogas e saúde na imprensa brasileira: uma análise de artigos publicados em jornais e revistas. Cad Saúde Pública 2003; 19:69-79.

30. Brasil. Lei no ${ }^{\circ} 11.705$, de 19 de junho de 2008. Altera a Lei ${ }^{\circ} .9 .503$, de 23 de setembro de 1997, que 'institui o Código de Trânsito Brasileiro', e a Lei no 9.294, de 15 de julho de 1996, que dispõe sobre as restrições ao uso e à propaganda de produtos fumígeros, bebidas alcoólicas, medicamentos, terapias e defensivos agrícolas, nos termos do $\$ 4^{\circ}$. do art. 220 da Constituição Federal, para inibir o consumo de bebida alcoólica por condutor de veículo automotor, e dá outras providências. Diário Oficial da União 2008; 20 jun.

31. Giovanucci E, Colditz G, Stampfer J, Rimm EB, Litin L, Sampson L, et al. The assessment of alcohol consumption by a simple self-administered questionnaire. Am J Epidemiol 1991; 133:810-7.

32. Laranjeira A, Pinsky I, Sanches M, Zaleski M, Caetano R. Alcohol use patterns among Brazilian adults. Rev Bras Psiquiatr 2010; 32:231-41.

Recebido em 15/Dez/2010

Versão final reapresentada em 04/Mai/2011

Aprovado em 26/Mai/2011 